\title{
A general quantum Laplace transform
}

\author{
Enas M. Shehata ${ }^{1 *}$ (D) Nashat Faried ${ }^{2}$ and Rasha M. El Zafarani ${ }^{2}$
}

${ }^{\text {*Correspondence: }}$ enasmohyi@yahoo.com

${ }^{1}$ Department of Mathematics and Computer Science, Faculty of

Science, Menoufia University, Shibin

El-Kom, Egypt

Full list of author information is

available at the end of the article

\section{Springer}

\begin{abstract}
In this paper, we introduce a general quantum Laplace transform $\mathcal{L}_{\beta}$ and some of its properties associated with the general quantum difference operator $D_{\beta} f(t)=(f(\beta(t))-f(t)) /(\beta(t)-t), \beta$ is a strictly increasing continuous function. In addition, we compute the $\beta$-Laplace transform of some fundamental functions. As application we solve some $\beta$-difference equations using the $\beta$-Laplace transform. Finally, we present the inverse $\beta$-Laplace transform $\mathcal{L}_{\beta}^{-1}$.

MSC: Primary 39A06; 39A13; 39A70; secondary 47B39

Keywords: General quantum difference operator; $\beta$-Laplace transform; Inverse $\beta$-Laplace transform; $\beta$-difference equations
\end{abstract}

\section{Introduction}

The Laplace transform in continuous and discrete cases has an essential role in applied mathematics and in mathematical physics, particularly in solving differential and difference equations, respectively. Recently, versions of Laplace transform in other calculi, such as $q$-calculus and time scale, were investigated, see [2-5]. The $q$-Laplace transform has a similar role in solving $q$-difference equations, see [1]. The general quantum difference operator $D_{\beta}$ is defined in [12] by

$$
D_{\beta} y(t)= \begin{cases}\frac{y(\beta(t))-y(t)}{\beta(t)-t}, & \beta(t) \neq t, \\ y^{\prime}(t), & \beta(t)=t,\end{cases}
$$

where the function $y$ is defined on an interval $I \subseteq \mathbb{R}$ and $\beta$ is a strictly increasing continuous general function, that is, $\beta(t) \in I$ for $t \in I$. The function $y$ is said to be $\beta$-differentiable if it is classic differentiable at the fixed points of the function $\beta$. Hamza et al. (2015) [12] established the calculus based on $D_{\beta}$ when $\beta$ has only one fixed point $s_{0} \in I$ that satisfies the inequality $\left(t-s_{0}\right)(\beta(t)-t) \leq 0$ for all $t \in I$, accordingly $\lim _{k \rightarrow \infty} \beta^{k}(t)=s_{0}$, $\beta^{k}(t):=\underbrace{\beta \circ \beta \circ \cdots \circ \beta}_{k \text {-times }}(t)$. Examples of this type are the Jackson $q$-difference operator with $\beta(t)=q t, 0<q<1, s_{0}=0$ and the Hahn difference operator with $\beta(t)=q t+\omega$, $0<q<1, \omega>0, s_{0}=\frac{\omega}{1-q}$. They mentioned also another type of $\beta$ when it has only one fixed point $s_{0} \in I$ and satisfies the inequality $\left(t-s_{0}\right)(\beta(t)-t) \geq 0$ for all $t \in I$; consequently, $\lim _{k \rightarrow \infty} \beta^{k}(t)=\infty$, for example, the backward Hahn difference operator with $\beta(t)=q t+\omega$,

(c) The Author(s) 2020. This article is licensed under a Creative Commons Attribution 4.0 International License, which permits use sharing, adaptation, distribution and reproduction in any medium or format, as long as you give appropriate credit to the original author(s) and the source, provide a link to the Creative Commons licence, and indicate if changes were made. The images or other third party material in this article are included in the article's Creative Commons licence, unless indicated otherwise in a credit line to the material. If material is not included in the article's Creative Commons licence and your intended use is not permitted by statutory regulation or exceeds the permitted use, you will need to obtain permission directly from the copyright holder. To view a copy of this licence, visit http://creativecommons.org/licenses/by/4.0/. 
$q>1, \omega>0$. A study of different types of the function $\beta$ according to the number of its fixed points, which can be basis for different calculi, was presented in [16]. In [13] some integral inequalities based on $D_{\beta}$ were introduced. The homogeneous second-order linear $\beta$-difference equations and the theory of $n$ th-order linear $\beta$-difference equations were studied in $[8,9]$. In addition, some properties of the quantum exponential functions in a Banach algebra were studied in [10]. Properties of the $\beta$-Lebesgue spaces were introduced in [6]. The $\beta$-difference operator $D_{\beta}$ and its calculus has applications in many areas in mathematics and physics such as the quantum variational calculus, the orthogonal polynomials, quantum mechanics, and scale of relativity, see [7, 14, 15].

In this paper we deduce a general quantum Laplace transform $\mathcal{L}_{\beta}$ associated with $D_{\beta}$, where $\beta$ has only one fixed point $s_{0} \in I$ with the inequality $\left(t-s_{0}\right)(\beta(t)-t) \leq 0$ for all $t \in I$, which will be useful in solving the $\beta$-difference equations. We organize this paper as follows: In Sect. 2 , we introduce the needed preliminaries from the $\beta$-calculus. In Sect. 3 , we present the $\beta$-regressive functions and define the " $\beta$-circle plus" $\oplus_{\beta}$ and the " $\beta$-circle minus" $\ominus_{\beta}$, and some associated relations. And then, we introduce the $\beta$-Laplace transform and some of its properties. Furthermore, we compute the $\beta$-Laplace transform of some fundamental functions. As application, we give two examples to solve some $\beta$-difference equations. Finally, we deduce the inverse $\beta$-Laplace transform $\mathcal{L}_{\beta}^{-1}$.

\section{Preliminaries}

In this section, we introduce some needed preliminaries from the $\beta$-calculus, where $\beta$ has only one fixed point $s_{0} \in I$ such that $\left(t-s_{0}\right)(\beta(t)-t) \leq 0$ for all $t \in I, \mathbb{X}$ is a Banach space.

Theorem 2.1 ([12]) Assume that $f: I \rightarrow \mathbb{X}$ and $g: I \rightarrow \mathbb{R}$ are $\beta$-differentiable functions on I. Then:

(i) The product $f g: I \rightarrow \mathbb{X}$ is $\beta$-differentiable at $t \in I$ and

$$
\begin{aligned}
D_{\beta}(f g)(t) & =\left(D_{\beta} f(t)\right) g(t)+f(\beta(t)) D_{\beta} g(t) \\
& =\left(D_{\beta} f(t)\right) g(\beta(t))+f(t) D_{\beta} g(t),
\end{aligned}
$$

(ii) $f / g$ is $\beta$-differentiable at $t \in I$ and

$$
\begin{aligned}
& \qquad D_{\beta}(f / g)(t)=\frac{\left(D_{\beta} f(t)\right) g(t)-f(t) D_{\beta} g(t)}{g(t) g(\beta(t))}, \\
& \text { provided that } g(t) g(\beta(t)) \neq 0 .
\end{aligned}
$$

Lemma $2.2([12])$ The following statements are true:

(i) The sequence of functions $\left\{\beta^{k}(t)\right\}_{k=0}^{\infty}$ converges uniformly to the constant function $\hat{\beta}(t):=s_{0}$ on every compact interval $J \subseteq I$ containing $s_{0}$.

(ii) The series $\sum_{k=0}^{\infty}\left|\beta^{k}(t)-\beta^{k+1}(t)\right|$ is uniformly convergent to $\left|t-s_{0}\right|$ on every compact interval $J \subseteq I$ containing $s_{0}$.

Theorem 2.3 ([12]) If $: I \rightarrow \mathbb{X}$ is continuous at $s_{0}$, then

(i) the sequence $\left\{f\left(\beta^{k}(t)\right)\right\}_{k=0}^{\infty}$ converges uniformly to $f\left(s_{0}\right)$,

(ii) the series $\sum_{k=0}^{\infty}\left\|\left(\beta^{k}(t)-\beta^{k+1}(t)\right) f\left(\beta^{k}(t)\right)\right\|$ is uniformly convergent on every compact interval $J \subseteq I$ containing $s_{0}$. 
Definition 2.4 ([12]) Let $f: I \rightarrow \mathbb{X}$ and $a, b \in I$. The $\beta$-integral of $f$ from $a$ to $b$ is defined by

$$
\int_{a}^{b} f(t) d_{\beta} t=\int_{s_{0}}^{b} f(t) d_{\beta} t-\int_{s_{0}}^{a} f(t) d_{\beta} t,
$$

where

$$
\int_{s_{0}}^{x} f(t) d_{\beta} t=\sum_{k=0}^{\infty}\left(\beta^{k}(x)-\beta^{k+1}(x)\right) f\left(\beta^{k}(x)\right), \quad x \in I,
$$

provided that the series converges at $x=a$ and $x=b . f$ is called $\beta$-integrable on $I$ if the series converges at $a$ and $b$ for all $a, b \in I$. Clearly, if $f$ is continuous at $s_{0} \in I$, then $f$ is $\beta$-integrable on $I$.

Theorem 2.5 ([12]) Assume that $f, g$ are $\beta$-differentiable functions on $I$ and $D_{\beta} f, D_{\beta} g$ are both continuous at $s_{0}$. Then

$$
\int_{a}^{b} f(t) D_{\beta} g(t) d_{\beta} t=f(b) g(b)-f(a) g(a)-\int_{a}^{b}\left(D_{\beta} f(t)\right) g(\beta(t)) d_{\beta} t, \quad a, b \in I .
$$

Here, at least one of the functions $f$ and $g$ is a real-valued function.

Definition 2.6 ([11]) The $\beta$-exponential functions $e_{p, \beta}(t)$ and $E_{p, \beta}(t)$ are defined by

$$
e_{p, \beta}(t)=\frac{1}{\prod_{k=0}^{\infty}\left[1-p\left(\beta^{k}(t)\right)\left(\beta^{k}(t)-\beta^{k+1}(t)\right)\right]}
$$

and

$$
E_{p, \beta}(t)=\prod_{k=0}^{\infty}\left[1+p\left(\beta^{k}(t)\right)\left(\beta^{k}(t)-\beta^{k+1}(t)\right)\right],
$$

where $p: I \rightarrow \mathbb{C}$ is a continuous function at $s_{0}$. Clearly, both products in (2.1) and (2.2) are convergent to a non-zero number for every $t \in I$, since $\sum_{k=0}^{\infty}\left|p\left(\beta^{k}(t)\right)\left(\beta^{k}(t)-\beta^{k+1}(t)\right)\right|$ is uniformly convergent.

Theorem 2.7 ([11]) The $\beta$-exponential functions $e_{p, \beta}(t)$ and $E_{p, \beta}(t)$ are the unique solutions of the $\beta$-initial value problems

$$
\begin{aligned}
& D_{\beta} y(t)=p(t) y(t), \quad y\left(s_{0}\right)=1, \\
& D_{\beta} y(t)=p(t) y(\beta(t)), \quad y\left(s_{0}\right)=1,
\end{aligned}
$$

respectively.

Definition 2.8 ([11]) The $\beta$-trigonometric functions are defined by

$$
\begin{aligned}
\cos _{p, \beta}(t) & =\frac{e_{i p, \beta}(t)+e_{-i p, \beta}(t)}{2}, \\
\sin _{p, \beta}(t) & =\frac{e_{i p, \beta}(t)-e_{-i p, \beta}(t)}{2 i} .
\end{aligned}
$$


Definition 2.9 ([11]) The $\beta$-hyperbolic functions are defined by

$$
\begin{aligned}
\cosh _{p, \beta}(t) & =\frac{e_{p, \beta}(t)+e_{-p, \beta}(t)}{2}, \\
\sinh _{p, \beta}(t) & =\frac{e_{p, \beta}(t)-e_{-p, \beta}(t)}{2} .
\end{aligned}
$$

Theorem 2.10 ([11]) Let $p: I \rightarrow \mathbb{C}$ be a continuous function at $s_{0}$. Then the following properties hold:

(i) $e_{p, \beta}(\beta(t))=[1+(\beta(t)-t) p(t)] e_{p, \beta}(t), t \in I$,

(ii) $D_{\beta}\left(\frac{1}{e_{p, \beta}(t)}\right)=\frac{-p(t)}{e_{p, \beta}(\beta(t))}$,

(iii) $\frac{1}{e_{p, \beta}(t)}$ is the unique solution of the first-order $\beta$-difference equation

$$
D_{\beta} y(t)=\frac{-p(t) e_{p, \beta}(t)}{e_{p, \beta}(\beta(t))} y(t), \quad y\left(s_{0}\right)=1
$$

Theorem 2.11 ([11]) Assume that $p, q: I \rightarrow \mathbb{C}$ are continuous functions at $s_{0} \in I$. The following properties are true:

(i) $\frac{1}{e_{p, \beta}(t)}=e_{-p /[1+(\beta(t)-t) p]}(t)$,

(ii) $e_{p, \beta}(t) e_{q, \beta}(t)=e_{p+q+(\beta(t)-t) p q}(t)$,

(iii) $e_{p, \beta}(t) / e_{q, \beta}(t)=e_{(p-q) /[1+(\beta(t)-t) q]}(t)$.

\section{Main results}

In this section, we present the $\beta$-regressive functions and define the " $\beta$-circle plus" $\oplus_{\beta}$ and the " $\beta$-circle minus" $\ominus_{\beta}$. We introduce the $\beta$-Laplace transform and some of its main properties. Furthermore, we compute the $\beta$-Laplace transform of the $\beta$-exponential and the $\beta$-trigonometric functions. As application, we give two examples to solve some $\beta$ difference equations. Finally, we deduce the inverse $\beta$-Laplace transform $\mathcal{L}_{\beta}^{-1}$.

\section{1 $\beta$-Regressive functions}

Definition 3.1 A function $p: I \rightarrow \mathbb{C}$ is said to be $\beta$-regressive on $I$ if $1+(\beta(t)-t) p(t) \neq 0$ for all $t \in I$.

We denote the set of all $\beta$-regressive functions $p: I \rightarrow \mathbb{C}$ and continuous at $s_{0}$ by $\mathcal{R}_{\beta}$, and the set of all $\beta$-regressive constants $z \in \mathbb{C}$ by $\mathcal{R}_{\beta}^{c}$.

Definition 3.2 Let $p, q \in \mathcal{R}_{\beta}$. Then we define $p \oplus_{\beta} q$, $\ominus_{\beta} p$, and $p \ominus_{\beta} q$ by

(i) $\left(p \oplus_{\beta} q\right)(t)=p(t)+q(t)+(\beta(t)-t) p(t) q(t), t \in I$,

(ii) $\left(\ominus_{\beta} p\right)(t)=\frac{-p(t)}{1+(\beta(t)-t) p(t)}, t \in I$,

(iii) $\left(p \ominus_{\beta} q\right)(t)=\left(p \oplus_{\beta}\left(\ominus_{\beta} q\right)\right)(t), t \in I$.

From the definition we conclude that $p \ominus_{\beta} p=0, \ominus_{\beta}\left(\ominus_{\beta} p\right)=p, \ominus_{\beta}\left(p \ominus_{\beta} q\right)=q \ominus_{\beta} p$, $\ominus_{\beta}\left(p \oplus_{\beta} q\right)=\left(\ominus_{\beta} p\right) \oplus_{\beta}\left(\ominus_{\beta} q\right)$, and $\left(\mathcal{R}_{\beta}, \oplus_{\beta}\right)$ form an abelian group.

Note that at $t=s_{0}, \oplus_{\beta}$ and $\ominus_{\beta}$ reduce to the classic addition and subtraction operations.

Theorem 3.3 Let $p, q \in \mathcal{R}_{\beta}, t \in I$. Then the following statements are true:

$$
\left(i_{1}\right) e_{\ominus_{\beta} p, \beta}(t)=\frac{1}{e_{p, \beta}(t)}=\prod_{k=0}^{\infty}\left[1-p\left(\beta^{k}(t)\right)\left(\beta^{k}(t)-\beta^{k+1}(t)\right)\right]=E_{-p, \beta}(t),
$$


$\left(i_{2}\right) e_{\ominus \beta p, \beta}(t)$ is the unique solution of the first-order $\beta$-difference equation

$$
D_{\beta} y(t)=\left(\ominus_{\beta} p\right)(t) y(t), \quad y\left(s_{0}\right)=1
$$

$\left(i_{3}\right)$

$$
\begin{aligned}
e_{\ominus_{\beta} p, \beta}(\beta(t)) & =\left[1+(\beta(t)-t)\left(\ominus_{\beta} p\right)(t)\right] e_{\ominus_{\beta}, \beta}(t)=\frac{e_{\ominus_{\beta} p, \beta}(t)}{1+(\beta(t)-t) p(t)} \\
& =-\frac{\left(\ominus_{\beta} p\right)(t)}{p(t)} e_{\ominus_{\beta}, \beta}(t)=-\frac{\left(\ominus_{\beta} p\right)(t)}{p(t) e_{p, \beta}(t)},
\end{aligned}
$$

$\left(i_{4}\right) D_{\beta}\left(e_{\ominus_{\beta} p, \beta}(t)\right)=\frac{\left(\ominus_{\beta} p\right)(t)}{e_{p, \beta}(t)}=\left(\ominus_{\beta} p\right)(t) e_{\ominus_{\beta} p, \beta}(t)=-p(t)\left[e_{\ominus_{\beta} p, \beta}(\beta(t))\right]$,

$\left(i_{5}\right) e_{p, \beta}(t) e_{q, \beta}(t)=e_{p \oplus_{\beta} q, \beta}(t)$,

(i, $) \frac{e_{p, \beta}(t)}{e_{q, \beta}(t)}=e_{p \ominus \beta q, \beta}(t)$.

\section{Proof}

$\left(i_{1}\right)$ Using Definition 2.6 and Theorem $2.11(i)$, we have

$$
\begin{aligned}
& e_{\ominus \beta p, \beta}(t)=e_{[1-(\beta(t)-t) p(t)]}^{-p(t)},(t)=\frac{1}{e_{p, \beta}(t)} \\
& =\prod_{k=0}^{\infty}\left[1-p\left(\beta^{k}(t)\right)\left(\beta^{k}(t)-\beta^{k+1}(t)\right)\right]=E_{-p, \beta}(t) \text {. }
\end{aligned}
$$

$\left(i_{2}\right)$ Since $\left(\ominus_{\beta} p\right)(t)=\frac{-p(t)}{1+(\beta(t)-t) p(t)}=\frac{-p(t) e_{p, \beta}(t)}{e_{p, \beta}(\beta(t))}$. Then equation (3.1) can be written as

$$
D_{\beta} y(t)=\frac{-p(t) e_{p, \beta}(t)}{e_{p, \beta}(\beta(t))} y(t), \quad y\left(s_{0}\right)=1 .
$$

By $\left(i_{1}\right)$ and Theorem 2.10 (iii), we get the desired result.

$\left(i_{3}\right)$ Using $\left(i_{1}\right),\left(i_{2}\right)$, we have

$$
\begin{aligned}
e_{\ominus_{\beta} p, \beta}(\beta(t)) & =e_{\ominus_{\beta} p, \beta}(t)+(\beta(t)-t)\left(D_{\beta} e_{\ominus_{\beta} p, \beta}(t)\right) \\
& =e_{\ominus_{\beta} p, \beta}(t)+(\beta(t)-t)\left(\ominus_{\beta} p\right)(t) e_{\ominus_{\beta} p, \beta}(t) \\
& =\left[1+(\beta(t)-t)\left(\ominus_{\beta} p\right)(t)\right] e_{\ominus_{\beta} p, \beta}(t) \\
& =\left[1-\frac{(\beta(t)-t) p(t)}{1+(\beta(t)-t) p(t)}\right] e_{\ominus_{\beta} p, \beta}(t) \\
& =\left[\frac{1}{1+(\beta(t)-t) p(t)}\right] e_{\ominus_{\beta} p, \beta}(t) \\
& =-\frac{\left(\ominus_{\beta} p\right)(t)}{p(t)} e_{\ominus \beta p, \beta}(t) \\
& =-\frac{\left(\ominus_{\beta} p\right)(t)}{p(t) e_{p, \beta}(t)} .
\end{aligned}
$$

$\left(i_{4}\right)$ From $\left(i_{1}\right)$ and Theorem 2.10, we get

$$
D_{\beta}\left(e_{\ominus_{\beta} p, \beta}(t)\right)=D_{\beta}\left(\frac{1}{e_{p, \beta}(t)}\right)=-\frac{p(t)}{e_{p, \beta}(\beta(t))}
$$




$$
\begin{aligned}
& =\frac{1}{e_{p, \beta}(t)} \frac{-p(t)}{[1+(\beta(t)-t) p(t)]} \\
& =\frac{1}{e_{p, \beta}(t)}\left(\ominus_{\beta} p\right)(t) \\
& =\left(\ominus_{\beta} p\right)(t) e_{\ominus \beta p, \beta}(t) .
\end{aligned}
$$

On the other hand, from $\left(i_{2}\right),\left(i_{3}\right)$

$$
-p(t)\left[e_{\ominus_{\beta} p, \beta}(\beta(t))\right]=\left(\ominus_{\beta} p\right)(t) e_{\ominus \beta p, \beta}(t)=D_{\beta}\left(e_{\ominus_{\beta} p, \beta}(t)\right) .
$$

$\left(i_{5}\right)$ From Theorem 2.11 (ii) and Definition 3.2, we get the desired result.

$\left(i_{6}\right)$ From $\left(i_{1}\right),\left(i_{5}\right)$, we get the result.

Lemma 3.4 Let $z, x \in R_{\beta}^{c}$ such that $z=x+i y$, where $z \in \mathbb{C}, x, y \in \mathbb{R}$. Then $\left|e_{\ominus_{\beta} z, \beta}(t)\right| \leq$ $e_{\ominus \beta} x, \beta(t)$.

Proof Using Theorem 2.11 (ii), we get

$$
e_{z, \beta}(t)=e_{(x+i y), \beta}(t)=e_{x, \beta}(t) e_{\frac{i y}{1+x(\beta(t)-t)}, \beta}(t) .
$$

So,

$$
\left|e_{z, \beta}(t)\right|=\left|e_{(x+i y), \beta}(t)\right| \geq e_{x, \beta}(t) .
$$

Then

$$
\left|\frac{1}{e_{z, \beta}(t)}\right|=\left|\frac{1}{e_{(x+i y), \beta}(t)}\right| \leq \frac{1}{e_{x, \beta}(t)} .
$$

Since $\frac{1}{e_{z, \beta}(t)}=e_{\ominus_{\beta} z, \beta}(t)$. Therefore,

$$
\left|e_{\ominus_{\beta} z, \beta}(t)\right| \leq e_{\ominus_{\beta} x, \beta}(t) .
$$

\subsection{The $\boldsymbol{\beta}$-Laplace transform}

In this section, let $\sup I=\infty, s_{0} \in I$. We assume that $z, \ominus_{\beta} z \in \mathcal{R}_{\beta}^{c}$ and hence $e_{\ominus \beta} z, \beta$ is well defined. Furthermore, we denote by $V\left(\left[s_{0}, \infty\right), \mathbb{C}\right)$ the set of $\beta$-integrable functions over each compact subinterval of $\left[s_{0}, \infty\right)$.

Definition 3.5 Let $\sup I=\infty, s_{0} \in I$ and $f(t)$ be continuous at $s_{0}$ on $\left[s_{0}, \infty\right)$. We define the improper $\beta$-integral by

$$
\begin{aligned}
\int_{s_{0}}^{\infty} f(t) d_{\beta} t & :=\lim _{b \rightarrow \infty} \int_{s_{0}}^{b} f(t) d_{\beta} t \\
& :=\lim _{b \rightarrow \infty} \sum_{k=0}^{\infty}\left(\beta^{k}(b)-\beta^{k+1}(b)\right) f\left(\beta^{k}(b)\right),
\end{aligned}
$$

provided this limit exists, and we say that the improper $\beta$-integral converges in this case. If this limit does not exist, then we say that the improper $\beta$-integral diverges. 
Definition 3.6 A function $f \in V\left(\left[s_{0}, \infty\right), \mathbb{C}\right)$ is said to be of exponential order $\lambda>0, \lambda \in \mathbb{R}$ if there exists a constant $M>0$ such that $|f(t)| \leq M e_{\lambda, \beta}(t)$ for all $t \in\left[s_{0}, \infty\right)$.

Definition 3.7 Suppose $f \in V\left(\left[s_{0}, \infty\right), \mathbb{C}\right)$. Then the Laplace transform of $f$ is defined by

$$
\mathcal{L}_{\beta}\{f(t)\}:=\int_{s_{0}}^{\infty} f(t) e_{\ominus_{\beta} z, \beta}(\beta(t)) d_{\beta} t
$$

for all $z \in \mathcal{R}_{\beta}^{c}$ for which the $\beta$-integral (3.3) exists.

Note that in the usual differential case, $\ominus_{\beta} z=-z, \beta(t)=t, e_{\ominus_{\beta} z \beta}(\beta(t))=e^{-z t}$, and (3.3) becomes the usual Laplace transform

$$
\mathcal{L}\{f(t)\}=\int_{0}^{\infty} f(t) e^{-z t} d t
$$

Moreover, in the case of $\beta(t)=q t, q \in(0,1)$, then $s_{0}=0, e_{\ominus \beta} z, \beta(\beta(t))=e_{\ominus q z, q}(q t)$, and we obtain the $q$-Laplace transform of the form

$$
\mathcal{L}_{q}\{f(t)\}=\int_{0}^{\infty} f(t) e_{\ominus_{q} z, q}(q t) d_{q} t
$$

see [4].

Theorem 3.8 Let $f \in V\left(\left[s_{0}, \infty\right), \mathbb{C}\right)$ be of exponential order $\lambda, z \in \mathcal{R}_{\beta}^{c}$ such that $z=x+i y$, $x, y \in \mathbb{R}$. Then the integral in the $\beta$-Laplace transform (3.3) converges absolutely for $|z|>\lambda$, provided that $\lim _{t \rightarrow \infty} e_{\lambda \Theta_{\beta} z, \beta}(t)=0$.

Proof Using Definition 3.6, Lemma 3.4, we get

$$
\begin{aligned}
\int_{s_{0}}^{\infty}\left|f(t) e_{\ominus_{\beta} z, \beta}(\beta(t))\right| d_{\beta} t & \leq \int_{s_{0}}^{\infty} M e_{\lambda, \beta}(t) e_{\ominus_{\beta} x, \beta}(\beta(t)) d_{\beta} t \\
& =\int_{s_{0}}^{\infty} \frac{M}{1+(\beta(t)-t) x} e_{\lambda, \beta}(t) e_{\ominus_{\beta} x, \beta}(t) d_{\beta} t \\
& =\int_{s_{0}}^{\infty} \frac{M}{1+(\beta(t)-t) x} e_{\left(\lambda \ominus_{\beta} x\right), \beta}(t) d_{\beta} t \\
& =\frac{M}{\lambda-x}\left[\int_{s_{0}}^{\infty} \frac{\lambda-x}{1+(\beta(t)-t) x} e_{\left(\lambda \Theta_{\beta} x\right), \beta}(t) d_{\beta} t\right] \\
& =\frac{M}{\lambda-x}\left[\int_{s_{0}}^{\infty}\left(\lambda \ominus_{\beta} x\right)(t) e_{\lambda \ominus_{\beta} x, \beta}(t) d_{\beta} t\right] \\
& =\frac{M}{\lambda-x}\left[\lim _{b \rightarrow \infty} \int_{s_{0}}^{b} D_{\beta}\left(e_{\lambda \ominus_{\beta} x, \beta}(t)\right) d_{\beta} t\right] \\
& =\frac{M}{x-\lambda} .
\end{aligned}
$$

Then $\mathcal{L}_{\beta}\{f(t)\}$ converges absolutely.

Example 3.9 Find the $\beta$-Laplace transform of $f(t) \equiv 1$. 
Sol. Using Theorem $3.3\left(i_{3}\right),\left(i_{4}\right)$, we have

$$
\begin{aligned}
\mathcal{L}_{\beta}\{1\} & =\int_{s_{0}}^{\infty} e_{\ominus_{\beta} z, \beta}(\beta(t)) d_{\beta} t \\
& =-\frac{1}{z}\left[\int_{s_{0}}^{\infty}\left(\ominus_{\beta} z\right) e_{\ominus_{\beta} z, \beta}(t) d_{\beta} t\right] \\
& =\frac{1}{z}\left[\lim _{b \rightarrow \infty} \int_{s_{0}}^{b} D_{\beta}\left(-e_{\ominus_{\beta} z, \beta}(t)\right) d_{\beta} t\right]=\frac{1}{z},
\end{aligned}
$$

provided that $\lim _{t \rightarrow \infty} e_{\ominus \beta} z, \beta(t)=0$.

Theorem 3.10 For $z, \lambda \in \mathcal{R}_{\beta}^{c}$,

$$
\mathcal{L}_{\beta}\left\{e_{\lambda, \beta}(t)\right\}=\frac{1}{z-\lambda},
$$

provided that $\lim _{t \rightarrow \infty} e_{\lambda \Theta_{\beta} z, \beta}(t)=0$.

Proof We find

$$
\begin{aligned}
\mathcal{L}_{\beta}\left\{e_{\lambda, \beta}(t)\right\} & =\int_{s_{0}}^{\infty} e_{\lambda, \beta}(t) e_{\ominus_{\beta} z, \beta}(\beta(t)) d_{\beta} t \\
& =\int_{s_{0}}^{\infty} \frac{1}{1+(\beta(t)-t) z} e_{\lambda, \beta}(t) e_{\ominus_{\beta} z, \beta}(t) d_{\beta} t \\
& =\int_{s_{0}}^{\infty} \frac{1}{1+(\beta(t)-t) z} e_{\left(\lambda \ominus_{\beta} z\right), \beta}(t) d_{\beta} t \\
& =\frac{1}{\lambda-z}\left[\int_{s_{0}}^{\infty} \frac{\lambda-z}{1+(\beta(t)-t) z} e_{\left(\lambda \ominus_{\beta} z\right), \beta}(t) d_{\beta} t\right] \\
& =\frac{1}{\lambda-z}\left[\int_{s_{0}}^{\infty}\left(\lambda \ominus_{\beta} z\right)(t) e_{\lambda \ominus_{\beta} z, \beta}(t) d_{\beta} t\right] \\
& =\frac{1}{\lambda-z}\left[\lim _{b \rightarrow \infty} \int_{s_{0}}^{b} D_{\beta}\left(e_{\lambda \ominus_{\beta} z, \beta}(t)\right) d_{\beta} t\right] \\
& =\frac{1}{z-\lambda},
\end{aligned}
$$

provided that $\lim _{t \rightarrow \infty} e_{\lambda \Theta_{\beta} z, \beta}(t)=0$.

Corollary 3.11 Let $\lambda, \mu, z \in \mathcal{R}_{\beta}^{c}$. Then

$$
\mathcal{L}_{\beta}\left\{e_{\frac{\lambda}{1+\mu(\beta(t)-t)}, \beta}(t) e_{\mu, \beta}(t)\right\}=\mathcal{L}_{\beta}\left\{e_{(\lambda+\mu), \beta}(t)\right\}=\frac{1}{z-(\lambda+\mu)},
$$

provided that $\lim _{t \rightarrow \infty} e_{(\lambda+\mu) \ominus_{\beta} z, \beta}(t)=0$.

Proof Using Theorem $3.3\left(i_{5}\right)$, and since

$$
\frac{\lambda}{1+\mu(\beta(t)-t)} \oplus_{\beta} \mu=\frac{\lambda}{1+\mu(\beta(t)-t)}+\mu+\frac{\lambda \mu(\beta(t)-t)}{1+\mu(\beta(t)-t)}
$$




$$
\begin{aligned}
& =\frac{\lambda+\mu+\mu^{2}(\beta(t)-t)+\lambda \mu(\beta(t)-t)}{1+\mu(\beta(t)-t)} \\
& =\frac{(\lambda+\mu)[1+\mu(\beta(t)-t)]}{1+\mu(\beta(t)-t)}=\lambda+\mu .
\end{aligned}
$$

Therefore, we have

$$
\mathcal{L}_{\beta}\left\{e_{\frac{\lambda}{1+\mu(\beta(t)-t)}, \beta}(t) e_{\mu, \beta}(t)\right\}=\mathcal{L}_{\beta}\left\{e_{(\lambda+\mu), \beta}(t)\right\}=\frac{1}{z-(\lambda+\mu)} .
$$

Theorem 3.12 (Linearity) Let $f, g \in V\left(\left[s_{0}, \infty\right), \mathbb{C}\right)$, and $c_{1}, c_{2}$ be constants. Then

$$
\mathcal{L}_{\beta}\left\{c_{1} f(t)+c_{2} g(t)\right\}=c_{1} \mathcal{L}_{\beta}\{f(t)\}+c_{2} \mathcal{L}_{\beta}\{g(t)\}
$$

Proof

$$
\begin{aligned}
\mathcal{L}_{\beta}\left\{c_{1} f(t)+c_{2} g(t)\right\} & =\int_{s_{0}}^{\infty}\left\{c_{1} f(t)+c_{2} g(t)\right\} e_{\ominus_{\beta} z, \beta}(\beta(t)) d_{\beta} t \\
& =\int_{s_{0}}^{\infty} c_{1} f(t) e_{\ominus_{\beta} z, \beta}(\beta(t)) d_{\beta} t+\int_{s_{0}}^{\infty} c_{2} g(t) e_{\ominus_{\beta} z, \beta}(\beta(t)) d_{\beta} t \\
& =c_{1} \mathcal{L}_{\beta}\{f(t)\}+c_{2} \mathcal{L}_{\beta}\{g(t)\} .
\end{aligned}
$$

Example 3.13 Find the $\beta$-Laplace transform of the following functions:

$$
\sin _{\lambda, \beta}(t), \quad \cos _{\lambda, \beta}(t), \quad \sinh _{\lambda, \beta}(t), \quad \text { and } \quad \cosh _{\lambda, \beta}(t) .
$$

Sol. By Definitions 2.8, 2.9 and since

$$
\mathcal{L}_{\beta}\left\{e_{\lambda, \beta}(t)\right\}=\frac{1}{z-\lambda}
$$

we have

$$
\begin{aligned}
\mathcal{L}_{\beta}\left\{\sin _{\lambda, \beta}(t)\right\} & =\mathcal{L}_{\beta}\left\{\frac{1}{2 i}\left[e_{i \lambda, \beta}(t)-e_{-i \lambda, \beta}(t)\right]\right\} \\
& =\frac{1}{2 i} \mathcal{L}_{\beta}\left\{e_{i \lambda, \beta}(t)\right\}-\frac{1}{2 i} \mathcal{L}_{\beta}\left\{e_{-i \lambda, \beta}(t)\right\} \\
& =\frac{1 / 2 i}{z-i \lambda}-\frac{1 / 2 i}{z+i \lambda}=\frac{\lambda}{z^{2}+\lambda^{2}}, \\
\mathcal{L}_{\beta}\left\{\cos _{\lambda, \beta}(t)\right\} & =\mathcal{L}_{\beta}\left\{\frac{1}{2}\left[e_{i \lambda, \beta}(t)+e_{-i \lambda, \beta}(t)\right]\right\} \\
& =\frac{1}{2} \mathcal{L}_{\beta}\left\{e_{i \lambda, \beta}(t)\right\}+\frac{1}{2} \mathcal{L}_{\beta}\left\{e_{-i \lambda, \beta}(t)\right\} \\
& =\frac{1 / 2}{z-i \lambda}+\frac{1 / 2}{z+i \lambda}=\frac{z}{z^{2}+\lambda^{2}},
\end{aligned}
$$




$$
\begin{aligned}
\mathcal{L}_{\beta}\left\{\sinh _{\lambda, \beta}(t)\right\} & =\mathcal{L}_{\beta}\left\{\frac{1}{2}\left[e_{\lambda, \beta}(t)-e_{-\lambda, \beta}(t)\right]\right\} \\
& =\frac{1}{2} \mathcal{L}_{\beta}\left\{e_{\lambda, \beta}(t)\right\}-\frac{1}{2} \mathcal{L}_{\beta}\left\{e_{-\lambda, \beta}(t)\right\} \\
& =\frac{1 / 2}{z-\lambda}-\frac{1 / 2}{z+\lambda}=\frac{\lambda}{z^{2}-\lambda^{2}},
\end{aligned}
$$

and

$$
\begin{aligned}
\mathcal{L}_{\beta}\left\{\cosh _{\lambda, \beta}(t)\right\} & =\mathcal{L}_{\beta}\left\{\frac{1}{2}\left[e_{\lambda, \beta}(t)+e_{-\lambda, \beta}(t)\right]\right\} \\
& =\frac{1}{2} \mathcal{L}_{\beta}\left\{e_{\lambda, \beta}(t)\right\}+\frac{1}{2} \mathcal{L}_{\beta}\left\{e_{-\lambda, \beta}(t)\right\} \\
& =\frac{1 / 2}{z-\lambda}+\frac{1 / 2}{z+\lambda}=\frac{z}{z^{2}-\lambda^{2}} .
\end{aligned}
$$

Theorem 3.14 ( $\beta$-Laplace transform of the $\beta$-derivative function) Let $f \in V\left(\left[s_{0}, \infty\right), \mathbb{C}\right)$ be a function of exponential order $\lambda$. Then

$$
\mathcal{L}_{\beta}\left\{D_{\beta} f(t)\right\}=z \mathcal{L}_{\beta}\{f(t)\}-f\left(s_{0}\right)
$$

provided that $\lim _{t \rightarrow \infty} f(t) e_{\ominus_{\beta} z, \beta}(t)=0$.

Proof Using Theorems 2.5, $3.3\left(i_{4}\right)$, we have

$$
\begin{aligned}
\mathcal{L}_{\beta}\left\{D_{\beta} f(t)\right\} & =\int_{s_{0}}^{\infty}\left[D_{\beta} f(t)\right] e_{\ominus_{\beta} z, \beta}(\beta(t)) d_{\beta} t \\
& =\lim _{b \rightarrow \infty} \int_{s_{0}}^{b} f(t) e_{\ominus \beta} z, \beta \\
& (t) d_{\beta} t-\int_{s_{0}}^{\infty}\left(\ominus_{\beta} z\right)(t) e_{\ominus \beta} z, \beta \\
& (t) f(t) d_{\beta} t \\
& =z\left[\int_{s_{0}}^{\infty} e_{\ominus_{\beta} z, \beta}(\beta(t)) f(t) d_{\beta} t\right]-f\left(s_{0}\right) \\
& =z \mathcal{L}_{\beta}\{f(t)\}-f\left(s_{0}\right) .
\end{aligned}
$$

Corollary 3.15 Let $f \in V\left(\left[s_{0}, \infty\right), \mathbb{C}\right)$ be a function of exponential order $\lambda$. Then, for any $n \in \mathbb{N}$, we have

$$
\mathcal{L}_{\beta}\left\{D_{\beta}^{n} f(t\}=z^{n} \mathcal{L}_{\beta}\{f(t)\}-\sum_{j=0}^{n-1} z^{n-1-j} D_{\beta}^{j} f\left(s_{0}\right) .\right.
$$

Proof As a consequence of Theorem 3.14 and using induction, we get

$$
\begin{gathered}
\mathcal{L}_{\beta}\left\{D_{\beta}^{2} f(t)\right\}=z\left[z \mathcal{L}_{\beta}\{f(t)\}-f\left(s_{0}\right)\right]-D_{\beta} f\left(s_{0}\right) \\
=z^{2} \mathcal{L}_{\beta}\{f(t)\}-z f\left(s_{0}\right)-D_{\beta} f\left(s_{0}\right), \\
\mathcal{L}_{\beta}\left\{D_{\beta}^{3} f(t)\right\}=z^{3} \mathcal{L}_{\beta}\{f(t)\}-z^{2} f\left(s_{0}\right)-z D_{\beta} f\left(s_{0}\right)-D_{\beta}^{2} f\left(s_{0}\right) .
\end{gathered}
$$

Assume that the corollary is true for $k \in \mathbb{N}$

$$
\mathcal{L}_{\beta}\left\{D_{\beta}^{k} f(t)\right\}=z^{k} \mathcal{L}_{\beta}\{f(t)\}-\sum_{m=0}^{k-1} z^{k-1-m} D_{\beta}^{m} f\left(s_{0}\right) .
$$


Then

$$
\begin{aligned}
\mathcal{L}_{\beta}\left\{D_{\beta}^{k+1} f(t)\right\} & =\mathcal{L}_{\beta}\left\{D_{\beta}\left(D_{\beta}^{k} f(t)\right)\right\} \\
& =z \mathcal{L}_{\beta}\left\{D_{\beta}^{k} f(t)\right\}-D_{\beta}^{k} f\left(s_{0}\right) \\
& =z\left[z^{k} \mathcal{L}_{\beta}\{f(t)\}-\sum_{m=0}^{k-1} z^{k-1-m} D_{\beta}^{m} f\left(s_{0}\right)\right]-D_{\beta}^{k} f\left(s_{0}\right) \\
& =z^{k+1} \mathcal{L}_{\beta}\{f(t)\}-\sum_{m=0}^{k-1} z^{k-m} D_{\beta}^{m} f\left(s_{0}\right)-D_{\beta}^{k} f\left(s_{0}\right) \\
& =z^{k+1} \mathcal{L}_{\beta}\{f(t)\}-\sum_{m=0}^{k} z^{k-m} D_{\beta}^{m} f\left(s_{0}\right) .
\end{aligned}
$$

Hence, the corollary holds for any $n \in \mathbb{N}$.

Example 3.16 Using the $\beta$-Laplace transform, find the solution of the $\beta$-initial value problem

$$
D_{\beta}^{2} y(t)+D_{\beta} y(t)-20 y(t)=0, \quad y\left(s_{0}\right)=2, D_{\beta} y\left(s_{0}\right)=-3 .
$$

Sol. By taking the $\beta$-Laplace transform and using equation (3.4), we have

$$
\begin{aligned}
0 & =z^{2} \mathcal{L}_{\beta}\{y(t)\}-2 z+3+\left[z \mathcal{L}_{\beta}\{y(t)\}-2\right]-20 \mathcal{L}_{\beta}\{y(t)\} \\
& =\left(z^{2}+z-20\right) \mathcal{L}_{\beta}\{y(t)\}-2 z,
\end{aligned}
$$

so that

$$
\mathcal{L}_{\beta}\{y(t)\}=\frac{2 z}{z^{2}+z-20}=\frac{10 / 9}{z+5}+\frac{8 / 9}{z-4},
$$

and hence

$$
y(t)=10 / 9 e_{-5, \beta}(t)+8 / 9 e_{4, \beta}(t) .
$$

Theorem 3.17 ( $\beta$-Laplace transform of the $\beta$-integral function) Let $f \in V\left(\left[s_{0}, \infty\right), \mathbb{C}\right)$ be a function of exponential order $\lambda$. Then

$$
\mathcal{L}_{\beta}\{F(t)\}=\frac{1}{z} \mathcal{L}_{\beta}\{f(t)\},
$$

where

$$
F(t):=\int_{s_{0}}^{t} f(\tau) d_{\beta} \tau
$$

provided that $\lim _{t \rightarrow \infty} F(t) e_{\ominus \beta} z, \beta(t)=0$. 
Proof Using Theorem 2.5, we have

$$
\begin{aligned}
\mathcal{L}_{\beta}\{F(t)\} & =\mathcal{L}_{\beta}\left\{\int_{s_{0}}^{t} f(\tau) d_{\beta} \tau\right\} \\
& =\int_{s_{0}}^{\infty} e_{\ominus_{\beta} z, \beta}(\beta(t)) F(t) d_{\beta} t \\
& =-\frac{1}{z}\left[\int_{s_{0}}^{\infty} F(t)\left[D_{\beta} e_{\ominus_{\beta} z, \beta}(\beta(t))\right] d_{\beta} t\right] \\
& =\frac{1}{z}\left[\int_{s_{0}}^{\infty} e_{\ominus_{\beta} z, \beta}(\beta(t)) f(t) d_{\beta} t\right]=\frac{1}{z} \mathcal{L}_{\beta}\{f(t)\},
\end{aligned}
$$

provided $\lim _{t \rightarrow \infty} F(t) e_{\ominus_{\beta} z, \beta}(t)=0$ holds.

Corollary 3.18 Assume $f \in V\left(\left[s_{0}, \infty\right), \mathbb{C}\right)$ and $\mathcal{L}_{\beta}\{f(t)\}=F(z)$. Then

$$
\mathcal{L}_{\beta}\left\{e_{\ominus_{\beta} \lambda, \beta}(\beta(t)) f(t)\right\}=F\left(z \oplus_{\beta} \lambda\right)
$$

Proof Using Theorem $3.3\left(i_{5}\right)$ and since $\ominus_{\beta}\left(z \oplus_{\beta} \lambda\right)=\left(\ominus_{\beta} \lambda\right) \oplus_{\beta}\left(\ominus_{\beta} z\right)$, we have

$$
e_{\ominus_{\beta} \lambda, \beta}(\beta(t)) e_{\ominus_{\beta} z, \beta}(\beta(t))=e_{\ominus_{\beta}\left(z \oplus_{\beta} \lambda\right), \beta}(\beta(t))
$$

Then

$$
\begin{aligned}
\mathcal{L}_{\beta}\left\{e_{\ominus_{\beta} \lambda, \beta}(\beta(t)) f(t)\right\} & =\int_{s_{0}}^{\infty} e_{\ominus_{\beta} z, \beta}(\beta(t))\left[e_{\ominus_{\beta} \lambda, \beta}(\beta(t)) f(t)\right] d_{\beta} t \\
& =\int_{s_{0}}^{\infty} e_{\ominus_{\beta}\left(z \oplus_{\beta} \lambda\right), \beta}(\beta(t)) f(t) d_{\beta} t \\
& =F\left(z \oplus_{\beta} \lambda\right) .
\end{aligned}
$$

Definition 3.19 Let $\lambda \in \mathcal{R}_{\beta}^{c}$. We define the functions $\psi_{k}: I \rightarrow \mathbb{C}$ for each $k \in \mathbb{N}_{0}$ recursively by taking $\psi_{0}(t):=1$, and

$$
\psi_{k+1}(t):=\int_{s_{0}}^{t} \frac{1}{1+\lambda(\beta(\tau)-\tau)} \psi_{k}(\tau) d_{\beta} \tau .
$$

Theorem 3.20 Let $\lambda \in \mathcal{R}_{\beta}^{c}$ and $n \in \mathbb{N}_{0}=\{0,1,2, \ldots\}$ be given. Then

$$
\mathcal{L}_{\beta}\left\{\psi_{n}(t) e_{\lambda, \beta}(t)\right\}=\frac{1}{(z-\lambda)^{n+1}},
$$

provided that

$$
\lim _{t \rightarrow \infty} \psi_{k}(t) e_{\lambda \ominus_{\beta} z, \beta}(t)=0 \quad \text { for each } k=0,1, \ldots, n
$$

Proof Using induction, for $n=0$, we have

$$
\mathcal{L}_{\beta}\left\{\psi_{0}(t) e_{\lambda, \beta}(t)\right\}=\mathcal{L}_{\beta}\left\{e_{\lambda, \beta}(t)\right\}=\frac{1}{z-\lambda} .
$$


For any $n \in \mathbb{N}$,

$$
\begin{aligned}
D_{\beta}\left(\psi_{n}(t)\right) & =D_{\beta}\left[\int_{s_{0}}^{t} \frac{1}{1+\lambda(\beta(\tau)-\tau)} \psi_{n-1}(\tau) d_{\beta} \tau\right] \\
& =\frac{1}{1+\lambda(\beta(t)-t)} \psi_{n-1}(t) .
\end{aligned}
$$

Suppose $\mathcal{L}_{\beta}\left\{\psi_{n-1}(t) e_{\lambda, \beta}(t)\right\}=\frac{1}{(z-\lambda)^{n}}$ for some $n \geq 1$. Then, by using Theorems 2.5, 3.3, we get

$$
\begin{aligned}
\mathcal{L}_{\beta} & \left\{\psi_{n}(t) e_{\lambda, \beta}(t)\right\} \\
= & \int_{s_{0}}^{\infty} \psi_{n}(t) e_{\lambda, \beta}(t) e_{\ominus_{\beta} z, \beta}(\beta(t)) d_{\beta} t \\
= & \int_{s_{0}}^{\infty} \psi_{n}(t)\left[1+(\beta(t)-t)\left(\ominus_{\beta} z\right)(t)\right] e_{\lambda \ominus_{\beta} z, \beta}(t) d_{\beta} t \\
= & \frac{1}{\lambda-z}\left[\int_{s_{0}}^{\infty} \psi_{n}(t)\left[\frac{\lambda-z}{1+z(\beta(t)-t)}\right] e_{\lambda \ominus_{\beta} z, \beta}(t) d_{\beta} t\right] \\
= & \frac{1}{\lambda-z}\left[\int_{s_{0}}^{\infty} \psi_{n}(t)\left(\lambda \ominus_{\beta} z\right)(t) e_{\lambda \Theta_{\beta} z, \beta}(t) d_{\beta} t\right] \\
= & \frac{1}{\lambda-z}\left[\lim _{b \rightarrow \infty} \int_{s_{0}}^{b} \psi_{n}(t) e_{\lambda \ominus_{\beta} z, \beta}(t) d_{\beta} t-\int_{s_{0}}^{\infty} D_{\beta}\left(\psi_{n}(t)\right) e_{\lambda \ominus_{\beta} z, \beta}(\beta(t)) d_{\beta} t\right] \\
= & \frac{1}{\lambda-z}\left[-\int_{s_{0}}^{\infty} D_{\beta}\left(\psi_{n}(t)\right) e_{\lambda \ominus_{\beta} z, \beta}(\beta(t)) d_{\beta} t\right] \\
= & \frac{1}{z-\lambda}\left[\int_{s_{0}}^{\infty} D_{\beta}\left(\psi_{n}(t)\right)\left[1+(\beta(t)-t)\left(\lambda \ominus_{\beta} z\right)(t)\right] e_{\lambda \ominus_{\beta} z, \beta}(t) d_{\beta} t\right] \\
= & \frac{1}{z-\lambda}\left[\int_{s_{0}}^{\infty}\left[\frac{\psi_{n-1}(t)}{1+\lambda(\beta(t)-t)}\right]\left[\frac{1+\lambda(\beta(t)-t)}{1+z(\beta(t)-t)}\right] e_{\lambda \ominus_{\beta} z, \beta}(t) d_{\beta} t\right] \\
= & \frac{1}{z-\lambda}\left[\int_{s_{0}}^{\infty} \psi_{n-1}(t) e_{\lambda, \beta}(t)\left[\frac{e_{\ominus_{\beta} z, \beta}(t)}{1+z(\beta(t)-t)}\right] d_{\beta} t\right] \\
= & \frac{1}{z-\lambda}\left[\int_{s_{0}}^{\infty} \psi_{n-1}(t) e_{\lambda, \beta}(t) e_{\ominus_{\beta} z, \beta}(\beta(t)) d_{\beta} t\right] \\
= & \frac{1}{z-\lambda}\left[\mathcal{L}_{\beta}\left\{\psi_{n-1}(t) e_{\lambda, \beta}(t)\right\}\right]=\frac{1}{(z-\lambda)^{n+1}} . \\
&
\end{aligned}
$$

Thus the desired result is satisfied for all $n \in \mathbb{N}$.

In the following theorem, we deduce the inverse $\beta$-Laplace transform $\mathcal{L}_{\beta}^{-1}$.

Theorem 3.21 For $z \in \mathcal{R}_{\beta}^{c}$ and $\lambda \neq 0$,

$$
\begin{aligned}
\mathcal{L}_{\beta}^{-1}\left\{\frac{1}{\left(z^{2}+\lambda^{2}\right)^{2}}\right\}= & \frac{\sin _{\lambda, \beta}(t)}{2 \lambda^{3}}-\frac{\cos _{\lambda, \beta}(t)}{2 \lambda^{2}} \int_{s_{0}}^{t} \frac{1}{1+\lambda^{2}(\beta(\tau)-\tau)^{2}} d_{\beta} \tau \\
& -\frac{\sin _{\lambda, \beta}(t)}{2 \lambda} \int_{s_{0}}^{t} \frac{(\beta(\tau)-\tau)}{1+\lambda^{2}(\beta(\tau)-\tau)^{2}} d_{\beta} \tau
\end{aligned}
$$


such that

$$
\lim _{t \rightarrow \infty} \psi_{k}(t) e_{i \lambda \ominus_{\beta} z, \beta}(t)=0 \quad \text { and } \quad \lim _{t \rightarrow \infty} \psi_{k}(t) e_{-i \lambda \Theta_{\beta} z, \beta}(t)=0, \quad k=0,1 .
$$

Proof Let $\lambda \neq 0$ be given. By the partial fraction

$$
\frac{1}{\left(z^{2}+\lambda^{2}\right)^{2}}=\frac{-1}{4 \lambda^{3} i(z+i \lambda)}-\frac{1}{4 \lambda^{2}(z+i \lambda)^{2}}+\frac{1}{4 \lambda^{3} i(z-i \lambda)}-\frac{1}{4 \lambda^{2}(z-i \lambda)^{2}},
$$

then taking the inverse $\beta$-Laplace transform and applying Theorem 3.10 and Theorem 3.20 , we obtain

$$
\begin{aligned}
\mathcal{L}_{\beta}^{-1}\left\{\frac{1}{\left(z^{2}+\lambda^{2}\right)^{2}}\right\}= & \frac{-1}{4 \lambda^{3} i} \mathcal{L}_{\beta}^{-1}\left\{\frac{1}{z+i \lambda}\right\}-\frac{1}{4 \lambda^{2}} \mathcal{L}_{\beta}^{-1}\left\{\frac{1}{(z+i \lambda)^{2}}\right\} \\
& +\frac{1}{4 \lambda^{3} i} \mathcal{L}_{\beta}^{-1}\left\{\frac{1}{z-i \lambda}\right\}-\frac{1}{4 \lambda^{2}} \mathcal{L}_{\beta}^{-1}\left\{\frac{1}{(z-i \lambda)^{2}}\right\} \\
= & \frac{-1}{4 \lambda^{3} i} e_{-i \lambda, \beta}(t)-\frac{1}{4 \lambda^{2}}\left[e_{-i \lambda, \beta}(t) \int_{s_{0}}^{t} \frac{1}{1-i \lambda(\beta(\tau)-\tau)} d_{\beta} \tau\right] \\
& +\frac{1}{4 \lambda^{3} i} e_{i \lambda, \beta}(t)-\frac{1}{4 \lambda^{2}}\left[e_{i \lambda, \beta}(t) \int_{s_{0}}^{t} \frac{1}{1+i \lambda(\beta(\tau)-\tau)} d_{\beta} \tau\right] \\
= & \frac{1}{2 \lambda^{3}}\left[\frac{e_{i \lambda, \beta}(t)-e_{-i \lambda, \beta}(t)}{2 i}\right]-\frac{1}{4 \lambda^{2}}\left[e_{-i \lambda, \beta}(t) \int_{s_{0}}^{t} \frac{1+i \lambda(\beta(\tau)-\tau)}{1+\lambda^{2}(\beta(\tau)-\tau)^{2}} d_{\beta} \tau\right. \\
& \left.+e_{i \lambda, \beta}(t) \int_{s_{0}}^{t} \frac{1-i \lambda(\beta(\tau)-\tau)}{1+\lambda^{2}(\beta(\tau)-\tau)^{2}} d_{\beta} \tau\right] \\
= & \frac{\sin _{\lambda, \beta}(t)}{2 \lambda^{3}}-\frac{1}{2 \lambda^{2}}\left[\frac{e_{-i \lambda, \beta}(t)+e_{i \lambda, \beta}(t)}{2}\right] \int_{s_{0}}^{t} \frac{1}{1+\lambda^{2}(\beta(\tau)-\tau)^{2}} d_{\beta} \tau \\
& -\frac{1}{2 \lambda}\left[\frac{e_{i \lambda, \beta}(t)-e_{-i \lambda, \beta}(t)}{2 i}\right] \int_{s_{0}}^{t} \frac{(\beta(\tau)-\tau)}{1+\lambda^{2}(\beta(\tau)-\tau)^{2}} d_{\beta} \tau \\
= & \frac{\sin _{\lambda, \beta}(t)}{2 \lambda^{3}}-\frac{\cos _{\lambda, \beta}(t)}{2 \lambda^{2}} \int_{s_{0}}^{t} \frac{1}{1+\lambda^{2}(\beta(\tau)-\tau)^{2}} d_{\beta} \tau \\
& -\frac{\sin _{\lambda, \beta}(t)}{2 \lambda} \int_{s_{0}}^{t} \frac{(\beta(\tau)-\tau)}{1+\lambda^{2}(\beta(\tau)-\tau)^{2}} d_{\beta} \tau .
\end{aligned}
$$

Corollary 3.22 Let $\lambda \neq 0, z \in \mathcal{R}_{\beta}^{c}$. The following relations hold:

(1) $\mathcal{L}_{\beta}^{-1}\left\{\frac{z}{\left(z^{2}+\lambda^{2}\right)^{2}}\right\}=\frac{\sin _{\lambda, \beta}(t)}{2 \lambda} \int_{s_{0}}^{t} \frac{1}{1+\lambda^{2}(\beta(\tau)-\tau)^{2}} d_{\beta} \tau-\frac{\cos _{\lambda, \beta}(t)}{2} \int_{s_{0}}^{t} \frac{(\beta(\tau)-\tau)}{1+\lambda^{2}(\beta(\tau)-\tau)^{2}} d_{\beta} \tau$.

(2) $\mathcal{L}_{\beta}^{-1}\left\{\frac{z^{2}}{\left(z^{2}+\lambda^{2}\right)^{2}}\right\}=\frac{\sin _{\lambda, \beta}(t)}{2 \lambda}+\frac{\cos _{\lambda, \beta}(t)}{2} \int_{s_{0}}^{t} \frac{1}{1+\lambda^{2}(\beta(\tau)-\tau)^{2}} d_{\beta} \tau+\frac{\lambda \sin _{\lambda, \beta}(t)}{2} \int_{s_{0}}^{t} \frac{(\beta(\tau)-\tau)}{1+\lambda^{2}(\beta(\tau)-\tau)^{2}} d_{\beta} \tau$.

(3) $\mathcal{L}_{\beta}^{-1}\left\{\frac{z^{3}}{\left(z^{2}+\lambda^{2}\right)^{2}}\right\}=\cos _{\lambda, \beta}(t)-\frac{\lambda \sin _{\lambda, \beta}(t)}{2} \int_{s_{0}}^{t} \frac{1}{1+\lambda^{2}(\beta(\tau)-\tau)^{2}} d_{\beta} \tau+\frac{\lambda^{2} \cos _{\lambda, \beta}(t)}{2} \int_{s_{0}}^{t} \frac{(\beta(\tau)-\tau)}{1+\lambda^{2}(\beta(\tau)-\tau)^{2}} d_{\beta} \tau$.

Example 3.23 Using the $\beta$-Laplace transform, find the solution of the $\beta$-initial value problem

$$
D_{\beta}^{2} y(t)-4 y(t)=t, \quad y\left(s_{0}\right)=1, D_{\beta} y\left(s_{0}\right)=2 .
$$

Sol. By applying the $\beta$-Laplace transform of equation (3.5), we get

$$
z^{2} y(z)-z y\left(s_{0}\right)-D_{\beta} y\left(s_{0}\right)-4 y(z)+4 y\left(s_{0}\right)=\frac{1}{z^{2}},
$$


and then

$$
y(z)=\frac{z^{3}-2 z^{2}+1}{z^{2}(z-2)(z+2)} .
$$

Therefore,

$$
\mathcal{L}_{\beta}^{-1}\{y(t)\}=\mathcal{L}_{\beta}^{-1}\left\{\frac{z^{3}-2 z^{2}+1}{z^{2}(z-2)(z+2)}\right\}
$$

Since

$$
\frac{z^{3}-2 z^{2}+1}{z^{2}(z-2)(z+2)}=\frac{-1 / 4}{z^{2}}+\frac{1 / 16}{z-2}+\frac{15 / 16}{z+2},
$$

then

$$
y(t)=-1 / 4 \mathcal{L}_{\beta}^{-1}\left\{\frac{1}{z^{2}}\right\}+1 / 16 \mathcal{L}_{\beta}^{-1}\left\{\frac{1}{z-2}\right\}+15 / 16 \mathcal{L}_{\beta}^{-1}\left\{\frac{1}{z+2}\right\} .
$$

Hence,

$$
y(t)=-1 / 4 t+1 / 16 e_{2, \beta}(t)+15 / 16 e_{-2, \beta}(t) .
$$

\section{Conclusion}

In this paper, a general quantum Laplace transform $\mathcal{L}_{\beta}$ associated with the general quantum difference operator $D_{\beta}$ and some of its properties were introduced. Moreover, the $\beta$-Laplace transform of some fundamental functions was computed. Finally, the inverse $\beta$-Laplace transform $\mathcal{L}_{\beta}^{-1}$ was presented.

Acknowledgements

The authors sincerely thank the referees for their valuable suggestions and comments.

Funding

Not applicable.

Availability of data and materials

Not applicable.

Competing interests

The authors declare that they have no competing interests.

Authors' contributions

All authors contributed equally and significantly in writing this article. All authors read and approved the final manuscript.

Author details

'Department of Mathematics and Computer Science, Faculty of Science, Menoufia University, Shibin El-Kom, Egypt.

${ }^{2}$ Department of Mathematics, Faculty of Science, Ain Shams University, Cairo, Egypt.

\section{Publisher's Note}

Springer Nature remains neutral with regard to jurisdictional claims in published maps and institutional affiliations.

Received: 14 May 2020 Accepted: 20 October 2020 Published online: 31 October 2020 


\section{References}

1. Abdi, W.H.: On certain q-difference equations and q-Laplace transform. Proc. Natl. Acad. Sci. India Sect. A 28, 1-15 (1962)

2. Bangerezako, G.: An introduction to q-difference equations. Bujumbura (2008)

3. Bastos, N.R.O., Mozyrska, D., Torres, D.F.M.: Fractional derivatives and integrals on time scales via the inverse generalized Laplace transform. Int. J. Math. Comput. 11, J11, 1-9 (2011)

4. Bohner, M., Guseinov, G.S.: The $h$-Laplace and q-Laplace transforms. J. Math. Anal. Appl. 365, 75-92 (2010)

5. Bohner, M., Peterson, A.: Dynamic Equations on Time Scales: An Introduction with Applications. Springer, Berlin (2001)

6. Cardoso, J.L.S.: Variations around a general quantum operator. Ramanujan J. (2020). https://doi.org/10.1007/s11139-019-00210-8

7. Cresson, J., Frederico, G., Torres, D.F.M.: Constants of motion for non-differentiable quantum variational problems. Topol. Methods Nonlinear Anal. 33, 217-231 (2009)

8. Faried, N., Shehata, E.M., El Zafarani, R.M.: On homogenous second order linear general quantum difference equations. J. Inequal. Appl. 2017, 198 (2017). https://doi.org/10.1186/s13660-017-1471-3

9. Faried, N., Shehata, E.M., El Zafarani, R.M.: Theory of $n$ th-order linear general quantum difference equations. Adv. Differ. Equ. 2018, 264 (2018). https://doi.org/10.1186/s13662-018-1715-7

10. Faried, N., Shehata, E.M., El Zafarani, R.M.: Quantum exponential functions in a Banach algebra. J. Fixed Point Theory Appl. 22, 22 (2020). https://doi.org/10.1007/s11784-020-0758-z

11. Hamza, A.E., Sarhan, A.M., Shehata, E.M.: Exponential, trigonometric and hyperbolic functions associated with a general quantum difference operator. Adv. Dyn. Syst. Appl. 12(1), 25-38 (2017)

12. Hamza, A.E., Sarhan, A.M., Shehata, E.M., Aldowah, K.A.: A general quantum difference calculus. Adv. Differ. Equ. 2015 182 (2015). https://doi.org/10.1186/s13660-015-0518-3

13. Hamza, A.E., Shehata, E.M.: Some inequalities based on a general quantum difference operator. J. Inequal. Appl. 2015, 38 (2015). https://doi.org/10.1186/s13660-015-0566-y

14. Malinowska, A.B., Torres, D.F.M.: Quantum Variational Calculus. Briefs in Electrical and Computer Engineering-Control, Automation and Robotics. Springer, Berlin (2014)

15. Nottale, L.: Fractal Space-Time and Microphysics: Towards a Theory of Scale Relativity. World Scientific, Singapore (1993)

16. Sarhan, A.M., Shehata, E.M.: On the fixed points of certain types of functions for constructing associated calculi. J. Fixed Point Theory Appl. 20, 124 (2018). https://doi.org/10.1007/s11784-018-0602-x

\section{Submit your manuscript to a SpringerOpen ${ }^{\circ}$ journal and benefit from:}

- Convenient online submission

- Rigorous peer review

- Open access: articles freely available online

- High visibility within the field

- Retaining the copyright to your article

Submit your next manuscript at $\boldsymbol{s p r i n g e r o p e n . c o m ~}$ 